\title{
Estresse da equipe de enfermagem em cuidados paliativos no enfrentamento da COVID-19
}

Stress in palliative care nursing staff while coping with COVID-19 Estrés del equipo de enfermería de cuidados paliativos en el enfrentamiento a la COVID-19

Daianny Arrais de Oliveira da Cunha ${ }^{1,2}$ (D) https://orcid.org/0000-0003-2109-319x

Patrícia dos Santos Claro Fuly² (B) https://orcid.org/0000-0002-0644-6447

Alex Sandro de Azeredo Siqueira' ${ }^{1}$ id https://orcid.org/0000-0002-6678-4499

Fernanda Barcellos Santiago ${ }^{1}$ id https://orcid.org/0000-0001-7067-7234

Endi Evelin Ferraz Kirby ${ }^{1}$ ic https://orcid.org/0000-0002-6250-3186

Vanessa dos Santos Beserra ${ }^{1}$ id https://orcid.org/0000-0001-9841-0021

Luciene Miguel Lima Neves ${ }^{1}$ io https://orcid.org/0000-0001-7881-9233

Como citar:

Cunha DA, Fuly PS, Siqueira AS, Santiago

$F B$, Kirby EE, Beserra VS, et al. Estresse da equipe de enfermagem em cuidados paliativos

no enfrentamento da COVID-19. Acta Paul Enferm. 2021;34:eAPE001915.

DOI

http://dx.doi.org/10.37689/actaape/2021A0001915

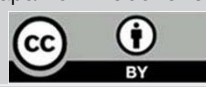

Descritores

Estresse ocupacional; Equipe de enfermagem; Cuidados paliativos; COVID-19; Pandemias

Keywords

Occupational stress; Nursing team; Palliative care; COVID-19; Pandemics

Descriptores

Estrés ocupacional; Equipo de enfermería; Cuidados paliativos; COVID-19; Pandemias

Submetido

22 de Julho de 2020

Aceito

26 de Julho de 2021

Autor correspondente

Daianny Arrais de Oliveira da Cunha E-mail: daoliveira@inca.gov.br

\section{Resumo}

Objetivo: Identificar a prevalência do estresse ocupacional dos profissionais de enfermagem que atuam em cuidados paliativos, durante a pandemia pelo SARS-CoV-2 e fatores sociodemográficos e ocupacionais associados.

Métodos: Trata-se de um estudo transversal, de abordagem quantitativa, realizado em uma unidade de atendimento a pacientes em cuidados paliativos oncológicos exclusivos, de um hospital oncológico público de referência nacional. A coleta de dados foi realizada entre os meses de abril e maio de 2020, utilizando dois instrumentos. Na análise dos dados foi utilizado o modelo de regressão de Poisson, com variância robusta.

Resultados: A amostra foi composta por 71 profissionais, que atuaram no atendimento à pacientes suspeitos ou com diagnóstico confirmado de COVID-19. 0 desfecho médio/alto nível de estresse foi prevalente em $42,2 \%$ dos profissionais, com maior predominância entre enfermeiros (65,0\%), que exercem suas atividades no período diarista/diurno $(55,2 \%)$ e que atuam há mais de oito anos em cuidados paliativos $(45,1 \%)$. Apenas as variáveis "cargo" e "morar sozinho" apresentaram associação significativa ao estresse médio/alto.

Conclusão: Diante dos resultados é importante que as instituições busquem medidas por meio de intervenções psicológicas e ocupacionais que possam reduzir os impactos mentais gerados pela atuação durante a COVID-19. Sugere-se maior estresse entre os enfermeiros, pois se envolvem em questões assistenciais e burocráticas, o que aumenta sua responsabilidade perante a equipe, e aos profissionais que residem sozinhos devido aos impactos gerados pelo isolamento e falta de apoio familiar próximo.

\section{Abstract}

Objective: To identify the prevalence of occupational stress among nursing professionals working in palliative care during the SARS-CoV-2 pandemic, and associated sociodemographic and occupational factors.

Methods: This was a cross-sectional study with a quantitative approach, conducted in an exclusive oncologic palliative care unit of a public oncology hospital of national reference. Data collection was performed between April and May of 2020, using two instruments. The Poisson regression model with robust variance was used for data analysis.

Results: The sample was composed of 71 professionals, working with patients suspected of or with a confirmed diagnosis of COVID-19. The medium/high level of stress outcome was prevalent in $42.2 \%$ of professionals, with higher prevalence among nurses (65.0\%), who worked during the day shift (55.2\%) and who had worked for more than eight years in palliative care (45.1\%). Only the variables "position" and "living alone" showed a significant association with medium/high stress. 
Conclusion: According the results, institutions must develop psychological and occupational interventions that can reduce the mental impact generated by nursing work during COVID-19. Increased stress was found among nurses, as they are involved in care and bureaucratic issues, which adds to their responsibility to the team, and among professionals who lived alone, due to impact generated by isolation and lack of close family support.

\section{Resumen}

Objetivo: Identificar la prevalencia del estrés laboral de los profesionales de enfermería que actúan en cuidados paliativos, durante la pandemia del SARSCoV-2 y factores sociodemográficos y laborales asociados.

Métodos: Se trata de un estudio transversal, de enfoque cuantitativo, realizado en una unidad de atención a pacientes en cuidados paliativos oncológicos exclusivos, de un hospital oncológico público de referencia nacional. La recopilación de datos fue realizada entre los meses de abril y mayo de 2020, mediante la utilización de dos instrumentos. En el análisis de los datos se utilizó el modelo de regresión de Poisson, con varianza robusta.

Resultados: La muestra estuvo compuesta por 71 profesionales que actuaban en la atención a pacientes con sospecha 0 diagnóstico confirmado de COVID-19. El resultado de nivel de estrés medio/alto fue prevalente en el 42,2 \% de los profesionales, con una mayor predominancia entre enfermeros $(65,0 \%)$, que ejercen sus actividades en el período diurno $(55,2 \%)$ y que actúan hace más de ocho años en cuidados paliativos (45,1\%). Solamente las variables "cargo" y "vivir solo" presentaron una asociación significativa con el estrés medio/alto.

Conclusión: Ante los resultados obtenidos, es importante que las instituciones busquen medidas a través de intervenciones psicológicas y laborales para reducir los impactos mentales generados por el trabajo durante la COVID-19. Se sugiere que existe mayor estrés entre los enfermeros, ya que están involucrados en cuestiones asistenciales y burocráticas, lo que aumenta su responsabilidad ante el equipo, y los profesionales que viven solos debido a los impactos generados por el aislamiento y la falta de apoyo familiar cercano.

\section{Introdução}

Em dezembro de 2019 o mundo iniciou o enfrentamento ao surto de uma doença infecciosa causada por um novo coronavírus (SARS-CoV-2) denominada COVID-19, que apresentou características de contágio exponencial sendo declarada em março, uma pandemia pela Organização Mundial de Saúde..$^{(1)}$

Em 7 de julho de 2020, a OMS informou um total de 1.500 .302 casos confirmados pelo mundo e 535.759 mortes foram informadas. No Brasil haviam 1.603.055 casos confirmados e um total de 64.867 mortes pela COVID-19..$^{(2)}$

Estudos relacionados a epidemias anteriores, ${ }^{(3,4)}$ como a Síndrome Respiratória Aguda Grave (SARS) em 2003 e a gripe H1N1 em 2009 apresentam resultados que apontam significativo impacto psicológico na comunidade, em função do medo e pânico relacionados à doença. Assim como os profissionais de saúde apresentaram relatos de extrema vulnerabilidade, sintomas somáticos e cognitivos de ansiedade e incertezas quanto aos riscos a que estavam expostos e, principalmente, ameaça à vida. ${ }^{(5)}$

Outros fatores estão associados a maior estresse, fadiga física e mental como o uso de equipamentos de proteção individual por longos períodos, ocasionando dificuldades respiratórias e limitando o acesso ao banheiro e à água. Associado a estes fatores, o excesso de informaçóes e reportagens da mídia são considerados estressores potenciais. ${ }^{(6)}$
Estudo recente realizado em um hospital chinês, referência no atendimento aos infectados pela COVID-19, revelou alta incidência de transtornos de ansiedade e estresse entre a equipe de saúde, sendo maior entre enfermeiros do que médicos $(26,8 \%$ vs $14,2 \%) .{ }^{(7)}$

Diante deste cenário, os profissionais de saúde que atuam em cuidados paliativos e vivenciam em sua rotina de trabalho processos dolorosos de final de vida, de sofrimento físico e existencial, podem exacerbar sintomas de acometimento mental. Principalmente quando a própria filosofia que embasa a atuação profissional está comprometida, visto que o isolamento social exigido neste período gera uma ruptura no relacionamento da equipe interdisciplinar com pacientes e familiares. ${ }^{(8)}$

A realidade aponta um importante limite na perspectiva da gestão de pessoas em cuidados paliativos, diante do número restrito de profissionais frente à maior demanda gerada por pacientes durante a pandemia. As mudanças necessárias na atuação paliativista, o aumento da procura dos serviços de cuidados paliativos, sobrecarga dos profissionais são fatores que podem inferir maior estresse na atuação destes profissionais. ${ }^{(9)}$

Diante da crescente demanda imposta pela pandemia no cenário dos cuidados paliativos e a lacuna no conhecimento acerca do estresse dos profissionais no cenário dos cuidados paliativos no enfrentamento da pandemia pelo SARS-CoV-2, surgem 
questóes. Qual a prevalência de médio/alto nível de estresse dos profissionais de enfermagem que atuam em cuidados paliativos durante a pandemia pelo novo coronavírus? Quais os fatores associados ao maior risco para níveis elevados de estresse ocupacional durante a pandemia?

Frente ao exposto, este estudo teve por objetivo: identificar a prevalência de estresse ocupacional dos profissionais de enfermagem que atuam em cuidados paliativos, durante a pandemia pelo SARSCoV-2 e fatores sociodemográficos e ocupacionais associados.

\section{Métodos}

Estudo transversal, de abordagem quantitativa, guiado pelo Strengthening the Reporting of Observational Studies in Epidemiology (STROBE). ${ }^{(10)}$ Foi realizado em um hospital público de referência nacional na prevenção, controle e tratamento do câncer e que apresenta uma unidade de atendimento a pacientes em cuidados paliativos oncológicos exclusivos.

Utilizou-se processo de amostragem não probabilística por conveniência. Foram elegíveis todos os trabalhadores integrantes da equipe de enfermagem que atuavam nas enfermarias do hospital e no pronto atendimento atendendo pacientes suspeitos de COVID-19. Foram excluídos os profissionais que estavam afastados do trabalho por algum tipo de licença.

A coleta de dados foi realizada entre os meses de abril e maio de 2020, utilizando dois instrumentos. $\mathrm{O}$ primeiro composto por um questionário de dados sociodemográficos e ocupacionais e o segundo trata-se da Escala de Estresse no Trabalho (EET), composta por 23 itens que formam um único fator ou escore. Os itens sáo avaliados de acordo com uma escala de concordância de cinco pontos do tipo likert (1 - discordo totalmente a 5 - concordo totalmente), onde os escores gerais podem variar de 23 a 115 pontos. ${ }^{(11)}$

Para análise das médias do escore foi adotado o ponto de corte de 2,5. Assim, média abaixo de 2,5 está relacionado a baixo nível de estresse, escore igual a 2,5 corresponde a médio ou considerável nível de estresse e médias acima de 2,5 configuram alto nível de estresse. ${ }^{(11)}$

A variável de desfecho (dependente) investigada no estudo foi à exposição ao estresse ocupacional (0 - Baixo; 1 - Médio/Alto) e as variáveis independentes (preditoras) foram divididas em dois grupos: as características sociodemográficas (sexo, idade, raça/ cor, nível de escolaridade, situação conjugal, resido com alguém, número de filhos, religião, remuneração, outra fonte de renda e presença de outro vínculo) e ocupacionais (cargo exercido, turno de trabalho, tempo de trabalho em cuidados paliativos).

Todas as análises foram realizadas utilizando o programa Stata 15.0 (Stata Corporation, College Station, Estados Unidos). Os dados foram transpostos para base de dados através do programa Microsoft Excel $^{\oplus}$ onde a análise dos dados foi realizada utilizando-se estatística descritiva. As variáveis categóricas foram analisadas em frequências absolutas e relativas e as contínuas em média e desvio padrão. Em seguida foi realizado o cálculo da prevalência do estresse ocupacional.

Para verificar a associação entre as variáveis preditoras e o desfecho foram estimadas razóes de prevalência e seus respectivos intervalos de confiança de 95\% por meio do modelo de regressão de Poisson com variância robusta. Em toda a análise foram considerados estatisticamente significativos valores de $p$ menores que 0,05 .

O projeto de pesquisa foi aprovado pelo Comitê de Ética em Pesquisa do Instituto Nacional de Câncer José Alencar Gomes da Silva, parecer número 3.992.180 e cada participante assinou o termo de consentimento livre e esclarecido. O estudo obedeceu às normas éticas previstas na Resolução 466/12, , $^{(2)}$ que dispóem dos aspectos éticos da pesquisa envolvendo seres humanos (CAAE: 30684120.0.0000.5274).

\section{Resultados}

Com base nos critérios do estudo e composição da amostra $(n=71)$ prevaleceram participantes do sexo feminino $(80,6 \%)$, com média de idade de 40,5 anos $( \pm 7,13)$, variação de 28 a 54 anos, casados 
$(69,0 \%)$ e de cor parda $(49,3 \%)$. Apenas $2,8 \%$ residem sozinhos e 19,7\% não tem filhos. Dos 79 profissionais que atendiam os critérios de inclusão, oito (10\%) recusaram a participação, sendo a amostra constituída por $71(89,8 \%)$ profissionais. A amostra foi composta em sua maioria por técnicos de enfermagem $(71,8 \%)$, com renda mensal entre nove e 12 salários mínimos $(53,5 \%)$ que não possuem outro vínculo empregatício $(59,2 \%)$. O tempo médio de experiência em cuidados paliativos foi de 10,2 anos $( \pm 4,63)$ com formação de nível superior $(39,4 \%)$ e predominantes no turno de trabalho diurno (40,8\%). O desfecho médio/alto nível de estresse foi prevalente em 42,2\% dos profissionais. Maior nível de estresse foi prevalente entre profissionais do sexo feminino $(45,6 \%)$, com idade até 40 anos $(43,9 \%)$, autodeclarados não brancos $(46,7 \%)$, pós graduados $(51,8 \%)$, solteiros, divorciados ou viúvos $(50,0 \%)$, que residiam sozinhos $(100,0 \%)$, sem filhos $(42,9 \%)$, adeptos de alguma religiāo $(44,8 \%)$ e com renda mensal entre nove e 12 salários mínimos $(47,4 \%)$, que apresentam outra fonte de ren$\mathrm{da}(50,0 \%)$ e sem vínculo empregatício adicional $(42,9 \%)$. $\mathrm{Na}$ análise bivariada (razão de prevalência ajustada), verificou-se alto nível de estresse para profissionais que residiam sozinhos $(\mathrm{p}=0,004)$ e pós graduados $(\mathrm{p}=0,24)$ (Tabela 1$)$.

O maior nível de estresse foi predominante entre enfermeiros $(65,0 \%)$, que exercem suas atividades no período diarista/diurno $(55,2 \%)$ e que atuam há mais de oito anos em cuidados paliativos $(45,1 \%)$. Na análise bivariada verificou-se maior nível de estresse entre os profissionais enfermeiros $(\mathrm{p}=0,01)$ que exercem suas atividades no período diarista/diurno $(\mathrm{p}=0,09)$ (Tabela 2).

Baseado nos dados da EET foi realizada a adequação de acordo com as dimensôes das quais se relacionam cada item da escala. Em seguida foram divididos de acordo com os escores que apresentaram o desfecho de interesse ( $1=$ médio/alto nível de estresse) em nível decrescente de pontuação. As dimensôes que pontuaram o maior número de itens relacionados ao desfecho foram "autonomia e controle" e "papéis e ambiente de trabalho". A tabela abaixo apresenta os itens com médias mensuradas para alto nível de estresse. Diante das médias ob-
Tabela 1. Prevalência e razão de prevalência do estresse de acordo com as características sociodemográficas

\begin{tabular}{|c|c|c|c|c|c|}
\hline Variáveis & Prevalência & $\mathrm{RP}_{\mathrm{b}}\left(\mathrm{IC}_{95 \%}\right)$ & $\mathrm{p}$-value & $\begin{array}{l}\mathrm{RP}^{*}{ }^{\mathrm{a}} \\
\left(\mathrm{IC}_{95 \%}{ }^{2}\right)\end{array}$ & $p$-value \\
\hline \multicolumn{6}{|l|}{ Sexo } \\
\hline Masculino & 28,6 & 1 & - & - & - \\
\hline Feminino & 45,6 & $\begin{array}{c}1,60(0,66 \text { - } \\
3,85)\end{array}$ & 0,30 & - & - \\
\hline \multicolumn{6}{|l|}{ Faixa etária (anos) } \\
\hline Até 40 & 43,9 & $\begin{array}{c}1,10(0,62- \\
1,93)\end{array}$ & 0,75 & - & - \\
\hline$\geq 40$ & 40,0 & 1 & - & - & - \\
\hline \multicolumn{6}{|l|}{ Raça/cor } \\
\hline Branca & 34,6 & 1 & - & - & - \\
\hline Não branca & 46,7 & $\begin{array}{c}1,35(0,73- \\
2,50)\end{array}$ & 0,34 & - & - \\
\hline \multicolumn{6}{|c|}{ Nível de escolaridade } \\
\hline Ensino médio & 37,5 & $\begin{array}{c}1,05(0,47- \\
2,36)\end{array}$ & 0,91 & - & - \\
\hline Nível superior & 35,7 & 1 & - & - & - \\
\hline Pós-graduação & 51,8 & $\begin{array}{c}1,45(0,78 \text { - } \\
2,70)\end{array}$ & 0,24 & - & - \\
\hline \multicolumn{6}{|l|}{ Situação conjugal } \\
\hline Casado & 38,8 & 1 & - & - & - \\
\hline Não casado & 50,0 & $\begin{array}{c}1,29(0,74- \\
2,23)\end{array}$ & 0,36 & - & - \\
\hline \multicolumn{6}{|l|}{ Reside com alguém } \\
\hline Sim & 40,6 & 1 & - & - & - \\
\hline Não & 100 & $\begin{array}{c}2,46(1,85- \\
3,28)\end{array}$ & $<0,001$ & $\begin{array}{c}2,12(1,28 \\
-3,54)\end{array}$ & 0,004 \\
\hline \multicolumn{6}{|l|}{ Tem filhos } \\
\hline Sim & 42,1 & 1 & - & - & - \\
\hline Não & 42,9 & $\begin{array}{c}1,02(0,51 \text { - } \\
2,01)\end{array}$ & 0,96 & - & - \\
\hline \multicolumn{6}{|l|}{ Religião } \\
\hline Sim & 44,8 & $\begin{array}{c}1,46(0,61 \text { - } \\
3,48)\end{array}$ & 0,40 & - & - \\
\hline Não & 30,8 & 1 & - & - & - \\
\hline \multicolumn{6}{|l|}{$\begin{array}{l}\text { Remuneração } \\
\text { recebida (SM) }\end{array}$} \\
\hline 6 a 9 & 42,1 & $\begin{array}{c}1,47(0,55- \\
3,96)\end{array}$ & 0,44 & - & - \\
\hline 9 a 12 & 47,4 & $\begin{array}{c}1,66(0,67- \\
4,08)\end{array}$ & 0,27 & - & - \\
\hline Acima de 12 & 28,6 & 1 & - & - & - \\
\hline \multicolumn{6}{|c|}{ Outra fonte de renda } \\
\hline Sim & 50,0 & $\begin{array}{c}1,26(0,71 \text { - } \\
2,24)\end{array}$ & 0,43 & - & - \\
\hline Não & 39,6 & 1 & - & - & - \\
\hline \multicolumn{6}{|l|}{ Outro vínculo } \\
\hline $\mathrm{Sim}$ & 41,4 & 1 & - & - & - \\
\hline Não & 42,9 & $\begin{array}{c}1,04(0,59- \\
1,81)\end{array}$ & 0,90 & - & - \\
\hline Prevalência & 42,2 & $30,6-54,6$ & - & & \\
\hline
\end{tabular}

RPb - razão de prevalência bruta; RPa - razão de prevalência ajustada; IC - intervalo de confiança

tidas o cenário ocupacional apresentou média de $2,51( \pm 0,75)$ caracterizando o alto nível de estresse ocupacional (Tabela 3). 
Tabela 2. Prevalência e razão de prevalência do estresse de acordo com as características ocupacionais

\begin{tabular}{|c|c|c|c|c|c|}
\hline Variáveis & Prevalência & $\operatorname{RPb}\left(\mathbf{I C}_{95 \%}\right)$ & $\mathrm{p}$-value & $\begin{array}{l}\mathrm{RP}^{* a} \mathrm{a} \\
\left(\mathrm{IC}_{95 \%}\right)\end{array}$ & $p$-value \\
\hline \multicolumn{6}{|l|}{ Cargo exercido } \\
\hline Enfermeiro & 65,0 & $\begin{array}{c}1,95(1,17 \\
-3,24)\end{array}$ & 0,01 & $\begin{array}{c}1,89(1,14 \\
-3,13)\end{array}$ & 0,01 \\
\hline Técnico de enfermagem & 33,3 & 1 & - & - & - \\
\hline \multicolumn{6}{|l|}{ Turno de trabalho } \\
\hline Diarista/Diurno & 55,2 & $\begin{array}{c}1,93(0,91 \\
-4,12)\end{array}$ & 0,09 & - & - \\
\hline Noturno & 28,6 & 1 & - & - & - \\
\hline Diurno/Noturno & 38,1 & $\begin{array}{c}1,33(0,56 \\
-3,20)\end{array}$ & 0,519 & - & - \\
\hline \multicolumn{6}{|l|}{$\begin{array}{l}\text { Tempo de trabalho em } \\
\text { CP (em anos) }\end{array}$} \\
\hline Até 8 & 35,0 & 1 & - & - & - \\
\hline Acima de 8 & 45,1 & $\begin{array}{c}1,29(0,66 \\
-2,53)\end{array}$ & 0,46 & - & - \\
\hline
\end{tabular}

$\mathrm{RPb}$ - razão de prevalência bruta; $\mathrm{RPa}$ - razão de prevalência ajustada; IC- intervalo de confiança. (*) Modelo final $=$ reside com alguém + cargo exercido

Tabela 3. Média dos escores avaliados para médio/alto nível de estresse no trabalho de acordo com as dimensões da Escala de Estresse no Trabalho

\begin{tabular}{|c|c|}
\hline Variáveis da escala de estresse no trabalho & $\begin{array}{l}\text { Score }(x / \text { Desvio- } \\
\text { padrão) }\end{array}$ \\
\hline \multicolumn{2}{|l|}{ Dimensão autonomia e controle } \\
\hline $\begin{array}{l}1 \text { - A forma como as tarefas são distribuídas em minha área tem me } \\
\text { deixado nervoso. }\end{array}$ & $3,0( \pm 1,1)$ \\
\hline $\begin{array}{l}22 \text { - } 0 \text { tempo insuficiente para realizar meu volume de trabalho } \\
\text { deixa-me nervoso. }\end{array}$ & $2,8( \pm 1,3)$ \\
\hline 2 - 0 tipo de controle existente no meu trabalho me irrita. & $2,8( \pm 1,0)$ \\
\hline $\begin{array}{l}13 \text { - Tenho me sentido incomodado com a deficiência nos } \\
\text { treinamentos para capacitação profissional. }\end{array}$ & $2,7( \pm 1,2)$ \\
\hline $\begin{array}{l}3 \text { - A falta de autonomia na execução do meu trabalho tem sido } \\
\text { desgastante. }\end{array}$ & $2,6( \pm 1,2)$ \\
\hline \multicolumn{2}{|l|}{ Dimensão papéis e ambiente de trabalho } \\
\hline $\begin{array}{l}9 \text { - Sinto-me incomodado por ter que realizar tarefas que estão além } \\
\text { de minha capacidade. }\end{array}$ & $3,2( \pm 1,3)$ \\
\hline $\begin{array}{l}10 \text { - Fico de mau humor por ter que realizar tarefas que estão além } \\
\text { de minha capacidade. }\end{array}$ & $3,2( \pm 1,2)$ \\
\hline $\begin{array}{l}5 \text { - Sinto-me irritado com a deficiência na divulgação de informações } \\
\text { sobre decisões do serviço. }\end{array}$ & $3,1( \pm 1,1)$ \\
\hline $\begin{array}{l}19 \text { - A falta de compreensão sobre quais são minhas } \\
\text { responsabilidades neste trabalho tem me causado irritação. }\end{array}$ & $2,6( \pm 1,2)$ \\
\hline \multicolumn{2}{|l|}{ Dimensão relacionamento com o chefe } \\
\hline $\begin{array}{l}12 \text { - Fico irritado com discriminação/ favoritismo no meu ambiente } \\
\text { de trabalho. }\end{array}$ & $3,1( \pm 1,1)$ \\
\hline 15 - Fico irritado por ser pouco valorizado por meus superiores. & $2,6( \pm 1,3)$ \\
\hline \multicolumn{2}{|l|}{ Dimensão crescimento e valorização } \\
\hline $\begin{array}{l}16 \text { - As poucas perspectivas de crescimento na carreira têm me } \\
\text { deixado angustiado. }\end{array}$ & $2,7( \pm 1,3)$ \\
\hline
\end{tabular}

\section{Discussão}

Os profissionais de enfermagem podem experimentar altos níveis de estresse pois os mesmos são responsáveis diretos pelo cuidado prestado ao paciente, gerenciamento do setor hospitalar e ainda atividades administrativas e burocráticas de diferentes níveis de complexidade. ${ }^{(13)}$ Assim a enfermagem é considerada uma das profissóes mais expostas ao risco de adoecimento físico e mental dentro das instituições, por enfrentarem condições de trabalho inadequadas, sobrecarga de trabalho diante de dimensionamento inadequado, ambiente insalubre e tarefas de repetição. ${ }^{(14)}$

Ponto importante a ser destacado diz respeito ao estresse ocupacional específico dos profissionais que atuam em oncologia e mais especificamente em cuidados paliativos. Cuidar de pacientes com prognósticos sombrios, sem possibilidade de cura atual, que apresentam sintomas de difícil controle pela própria evoluçáo da doença, encarar o processo ativo de morte dos pacientes e validar a sua própria consciência de finitude, são elementos que tornam esses profissionais vulneráveis ao desenvolvimento de estresse ocupacional seguidos de diversos tipos de sofrimento psicológico. ${ }^{(15)}$

Existem estudos que evidenciam a importância dos profissionais que atuam em cuidados paliativos em meio a grandes desastres e epidemias/pandemias como a vivenciada atualmente. No entanto, não foram encontrados estudos avaliando a saúde mental destes profissionais no enfrentamento da COVID-19. ${ }^{(16)}$

Avaliando o cenário do período da SARS, em 2003, um estudo identificou que $89 \%$ dos profissionais de saúde que estavam em situaçóes de alto risco, ou seja, na linha de frente de atendimento, relataram sintomas psicológicos, dentre eles o estresse ocupacional. Este estudo concluiu que o ambiente ocupacional foi caracterizado para alto nível de estresse ocupacional. $\mathrm{O}$ estresse médio/alto foi prevalente em $42,2 \%$ dos participantes. ${ }^{(17)}$

Já um recente estudo norte-americano ${ }^{(18)}$ evidenciou que mais da metade dos profissionais de saúde $(57,0 \%)$ apresentou resultado positivo para estresse agudo (com possibilidade de apresentar sintomas de transtorno de estresse pós traumático). $\mathrm{O}$ maior número de participantes $(\mathrm{n}=657)$ do estudo e número de instrumentos utilizados pode justificar a maior prevalência de estresse.

Tratando-se de profissionais que atuam em cuidados paliativos um estudo brasileiro anterior a pandemia avaliou 105 profissionais constatando níveis de estresse moderado/alto em 41,9\% dos investiga- 
dos. $\mathrm{O}$ que sugere que profissionais que atuam em cuidados paliativos experimentam chances maiores de desenvolver estresse ocupacional. ${ }^{(19)}$

$\mathrm{O}$ estresse foi prevalente entre profissionais do sexo feminino e com idade até 40 anos, sendo dado aproximado a um estudo chinês que avaliou 1257 profissionais, onde o estresse foi prevalente em $71,5 \%$ dos participantes, sendo maior entre enfermeiros do que entre os médicos (36,2\% vs 33,0\%). (20) Além disso, as mulheres tendem a ter maior atenção corporal e consciência dos sintomas físicos e psicológicos que os homens. ${ }^{(21)}$

Como nosso estudo avaliou especificamente a equipe de enfermagem encontramos prevalência maior entre os enfermeiros quando comparados aos técnicos de enfermagem $(65,0 \%$ vs $33,3 \%, \mathrm{p}=0,01)$. Diferença confirmada em um estudo realizado no sudeste do Brasil $^{(22)}(83,3 \%$ vs $69,0 \%, \mathrm{p}=0,16)$ que avaliou 124 profissionais, porém utilizando instrumento de coleta que avaliou o estresse em níveis diferentes do instrumento utilizado no presente estudo. $\mathrm{O}$ nível de responsabilidades exigido aos enfermeiros diante de suas tarefas assistenciais e gerenciais pode justificar tal achado.

Além do cargo ocupado (enfermeiros e técnicos de enfermagem) os profissionais que moram sozinhos apresentaram estresse significativo comparados aos que residiam com alguém (100,0\% vs 40,6\%), no entanto, apenas dois participantes estavam no grupo que residia sozinho, podendo caracterizar viés importante.

Diante dos escores medidos através da EET verificou-se que as dimensóes com mais itens pontuados para o desfecho foram "autonomia e controle" e "papéis e ambiente de trabalho". O comprometimento psicológico dos profissionais de saúde diante de uma epidemia pode ser complexo. As fontes de angústia podem incluir sentimentos de vulnerabilidade ou perda de controle, preocupaçóes com a própria saúde e de seus familiares, a disseminação do vírus, mudanças na rotina de trabalho e a necessidade de isolamento. ${ }^{(23)}$

Com base nos dados apresentados, entende-se que o estresse é uma alteração psicológica que impacta diretamente no cotidiano dos profissionais de enfermagem que atuam em cuidados paliativos.
Diante de períodos de crise como a pandemia que se instalou no final de 2019 a saúde mental destes profissionais fica seriamente comprometida, ocasionando possivelmente outros sintomas mentais e físicos. ${ }^{(24)}$ Desse modo estratégias institucionais podem contribuir para o equilíbrio na saúde mental dos profissionais que se encontram na linha de frente de cuidado, oferecendo mecanismos para a manutenção das necessidades humanas básicas. ${ }^{(25)}$

$\mathrm{O}$ estudo apresenta delineamento de corte transversal o que impossibilita a avaliação de causalidade bem como o acompanhamento do fenômeno estudado. Ainda, por ter sido adotada amostragem por conveniência esta pode não representar a realidade.

\section{Conclusão}

Diante dos resultados obtidos constata-se que as instituiçôes devem buscar medidas através de intervençôes psicológicas que possam reduzir os impactos mentais gerados pela atuação durante a COVID-19. O fortalecimento dos treinamentos, quantitativo suficiente de profissionais de enfermagem, tempo de descanso adequado, atualização das informaçóes mais recentes prontamente e o incentivo aos profissionais para compartilhar experiências clínicas e sentimentos pode auxiliar na redução das taxas de transtorno de estresse pós-traumático conforme experimentado em outras epidemias. Sugere-se maior estresse entre os enfermeiros, pois se envolvem em questóes não somente assistenciais, mas burocráticas, o que aumenta sua responsabilidade perante a equipe, e aos profissionais que residem sozinhos devido aos impactos gerados pelo isolamento e falta de apoio familiar próximo. Este estudo foi realizado durante o início do surto de COVID-19 no Brasil, quando o conhecimento acerca da pandemia era limitado e as informaçóes mudavam rapidamente. Posteriormente realizaremos nova investigação combinando outros instrumentos visando melhor entendimento sobre o impacto psicossocial que acomete os profissionais de enfermagem que atuam em cuidados paliativos durante a COVID-19. 


\section{Colaborações}

Cunha DAO, Fuly PSC, Siqueira ASA, Santiago FB, Kirby EEF, Beserra VS e Neves LML contribuíram com a concepçáa do estudo, análise e interpretação dos dados, redação do artigo, revisão crítica relevante do conteúdo intelectual e aprovação da versão final a ser publicada.

\section{Referências}

1. World Health Organization (WHO). WHO announces COVID-19 outbreak a pandemic. Genève: WHO; 2020 [cited 2020 July 7]. Available from: http://www.euro.who.int/en/health-topics/health-emergencies/ coronavirus-covid-19/news/news/2020/3/who-announces-covid-19outbreak-a-pandemic

2. World Health Organization (WHO). Coronavirus disease (COVID-19) situation reports. Genève: WHO; 2020 [cited 2020 July 7. Available from: www.who.int/emergencies/diseases/novel-coronavirus-2019/ situation-reports

3. Chong MY, Wang WC, Hsieh WC, Lee CY, Chiu NM, Yeh WC, et al. Psychological impact of severe acute respiratory syndrome on health workers in a tertiary hospital. Br J Psychiatry. 2004;185:127-33.

4. Goulia P, Mantas C, Dimitroula D, Mantis D, Hyphantis T. General hospital staff worries, perceived sufficiency of information and associated psychological distress during the A/H1N1 influenza pandemic. BMC Infect Dis. 2010;10:322.

5. Tsamakis K, Rizos E, Manolis AJ, Chaidou S, Kympouropoulos S, Spartalis E, et al. COVID-19 pandemic and its impact on mental health of healthcare professionals. Exp Ther Med. 2020;19(6):3451-3.

6. Chen Q, Liang M, Li Y, Guo J, Fei D, Wang L, et al. Mental health care for medical staff in China during the COVID-19 outbreak. Lancet Psychiatry. 2020;7(4):e15-e16. Erratum in: Lancet Psychiatry. 2020;7(5):e27.

7. Huang JZ, Han MF, Luo TD, Ren AK, Zhou XP. [Mental health survey of medical staff in a tertiary infectious disease hospital for COVID-19]. Zhonghua Lao Dong Wei Sheng Zhi Ye Bing Za Zhi. 2020;38(3):192195. Chinese.

8. Shalev D, Nakagawa S, Stroeh OM, Arbuckle MR, Rendleman R, Blinderman $C D$, et al. The creation of a psychiatry-palliative care liaison team: using psychiatrists to extend palliative care delivery and access during the COVID-19 crisis. J Pain Symptom Manage. 2020;60(3):e12-e16.

9. Vergano M, Bertolini G, Giannini A, Gristina GR, Livigni S, Mistraletti G, et al. Clinical ethics recommendations for the allocation of intensive care treatments in exceptional, resource-limited circumstances: the Italian perspective during the COVID-19 epidemic [Editorial]. Crit Care. 2020;24(1):165.

10. Cheng A, Kessler D, Mackinnon R, Chang TP, Nadkarni VM, Hunt EA, Duval-Arnould J, Lin Y, Cook DA, Pusic M, Hui J, Moher D, Egger M, Auerbach $\mathrm{M}$; International Network for Simulation-based Pediatric
Innovation, Research, and Education (INSPIRE) Reporting Guidelines Investigators. Reporting Guidelines for Health Care Simulation Research: Extensions to the CONSORT and STROBE Statements. Simul Healthc. 2016;11(4):238-48.

11. Paschoal T, Tamayo A. Validação da Escala de Estresse no Trabalho. Estudos Psicol. 2004;9(1):45-52.

12. Brasil. Ministério da Saúde. Conselho Nacional de Saúde. Resolução 466/12. Trata de pesquisas em seres humanos e atualiza a resolução 196. Brasília (DF): Ministério da Saúde; 2012 [citado 2012 Jun 13]. Disponivel: http://conselho.saude.gov.br/resolucoes/2012/Reso466. pdf

13. Scholze AR, Martins JT, Robazzi ML, Haddad MC, Galdino MJ, Ribeiro RP. Occupational stress and associated factors among nurses at public hospitals. Cogitare Enferm. 2017;22(3):e50238.

14. Cacciari P, Hadadd MC, Vannuchi MT, Dalmas JC. Estado de saúde de trabalhadores de enfermagem em readequação e readaptação funcional. Rev Bras Enferm. 2013;66(6):860-5.

15. Santos NA, Gomes SV, Rodrigues CM, Santos J, Passos JP. Estratégias de enfrentamento utilizadas pelos enfermeiros em cuidados paliativos oncológicos: revisão integrativa. Cogitare Enferm. 2016;21(3):1-7.

16. Rodin G, Zimmermann C, Rodin D, Al-Awamer A, Sullivan R, Chamberlain C. COVID-19, palliative care and public health. Eur $\mathrm{J}$ Cancer. 2020;136:95-8.

17. Chua SE, Cheung V, Cheung C, McAlonan GM, Wong JW, Cheung EP, et al. Psychological effects of the SARS outbreak in Hong Kong on highrisk health care workers. Can J Psychiatry. 2004;49(6):391-3.

18. Shechter A, Diaz F, Moise N, Anstey DE, Ye S, Agarwal S, et al. Psychological distress, coping behaviors, and preferences for support among New York healthcare workers during the COVID-19 pandemic. Gen Hosp Psychiatry. 2020;66:1-8.

19. Santos NA, Santos J, Silva V, Passos J. Estresse ocupacional na assistência de cuidados paliativos em oncologia. Cogitare Enferm. 2017;22(4):1-10.

20. Lai J, Ma S, Wang Y, Cai Z, Hu J, Wei N, et al. Factors associated with mental health outcomes among health care workers exposed to coronavirus disease 2019. JAMA Netw Open. 2020;3(3):e203976.

21. Jahrami H, BaHammam AS, AlGahtani H, Ebrahim A, Faris M, AlEid K, et al. The examination of sleep quality for frontline healthcare workers during the outbreak of COVID-19. Sleep Breath. 2021;25(1):503-11.

22. Santana LC, Ferreira LA, Santana LP. Estresse ocupacional em profissionais de enfermagem de um hospital universitário. Rev Bras Enferm. 2020;73(2):e20180997.

23. Wong TW, Yau JK, Chan CL, Kwong RS, Ho SM, Lau CC, et al. The psychological impact of severe acute respiratory syndrome outbreak on healthcare workers in emergency departments and how they cope. Eur J Emerg Med. 2005;12(1):13-8.

24. Chew NW, Lee GK, Tan BY, Jing M, Goh Y, Ngiam NJ, et al. A multinational, multicentre study on the psychological outcomes and associated physical symptoms amongst healthcare workers during COVID-19 outbreak. Brain Behav Immun. 2020;88:559-65.

25. Gonzalez A, Cervoni C, Lochner M, Marangio J, Stanley C, Marriott S. Supporting health care workers during the COVID-19 pandemic: Mental health support initiatives and lessons learned from an academic medical center. Psychol Trauma. 2020;12(S1):S168-S170. 